\title{
Applied Photochemistry - Light Controlled Perfume Release
}

\author{
Samuel Derrer ${ }^{\mathrm{a}}$, Felix Flachsmann ${ }^{\star a}$, Caroline Plessis $^{\mathrm{b}}$, and Melanie Stang ${ }^{\mathrm{c}}$
}

\begin{abstract}
Ambient light is one of the most suitable available trigger conditions for the release of covalently bound volatile odorants in home- and laundry-care applications. We report on three complementary classes of light-cleavable fragrance precursors, covering the controlled release of odorants with a wide range of functional groups. o-Hydroxy cinnamates 1 undergo a UV-induced double bond isomerization followed by transesterification to release coumarin and a fragrance alcohol of choice. $\alpha$-Alkoxyacetophenones 10 and $\alpha, \alpha$-dialkoxyacetophenones 12 undergo Norrish type II fragmentations upon UV-irradiation, thereby releasing one or two equivalents of fragrant aldehydes, respectively. Finally, photoexcited Xanthenoic esters 22 undergo fragmentation to release reactive acyl radicals, which further cyclize onto internal olefins to form perfumery lactones of various ring sizes.
\end{abstract}

Keywords: Fragrance precursor · Light cleavable · Norrish type II · Photoisomerization · Pro-perfume

\section{Introduction}

Improving perfume longevity is a prime research goal in the fragrance industry. In the world of perfumed consumer products, this implies mainly the prevention of losses of perfume during storage (premature evaporation, chemical degradation) and application (low affinity to substrate, washout in surfactant-rich aqueous phases and evaporation during the drying process). The physical and olfactory parameters which determine the longlastingness of each individual fragrance ingredient in a given application (referred to also as substantivity) differ widely across olfactory and structural classes. Numerous highly substantive compounds are found among woody, ambery and musky odorants, ${ }^{[1]}$ whereas molecules exhibiting floral, fruity, aldehydic or green notes, essential for transmitting freshness and cleanliness, are often relatively small,

\footnotetext{
${ }^{\star}$ Correspondence: Dr. F. Flachsmann ${ }^{a}$

Tel.: +41448242324

Fax: + 41448242926

E-Mail: felix.flachsmann@givaudan.com

aGivaudan Schweiz AG

Fragrance Research

Ueberlandstrasse 138

$\mathrm{CH}-8600$ Dübendorf

bCurrent address: Mane, 620 Route de Grasse,

06620 Le Bar-Sur-Loup, France

'Current address: Novartis Pharma AG,

Lichtstrasse 35, CH-4056 Basel
}

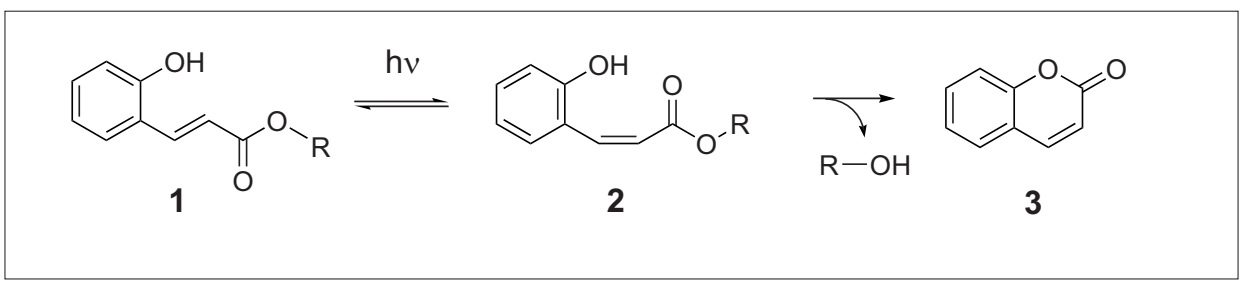

Scheme 1. Photoisomerization and lactonization of E-o-hydroxy cinnamates. Substituents on the aromatic rings are omitted for the sake of clarity.

volatile and sufficiently water-soluble to be lost in dilute aqueous applications.

In order to overcome this substantivity problem, the use of fragrance precursors (also referred to as profragrances or properfumes) has been envisaged, in which odorants are bound to a larger molecular backbone, from which they are slowly released via hydrolytic, enzymatic, thermal or photolytic cleavage mechanisms. For consumer product applications, light is an ideal physical trigger, since it can be easily excluded during storage and is generally present when perfume release is expected. In the following account, we describe three classes of fragrance precursors, in which photoexcitation leads directly to fragmentation according to a Norrish type II mechanism or, alternatively, produces unstable species, which ultimately break apart to release the fragrance molecules.

\section{2. o-Hydroxy Cinnamic Acid Esters}

In the course of our long-standing research program on sunscreen agents, we have gained considerable experience in the photochemical behavior of substituted cinnamates (e.g. ethyl hexyl methoxycinnamate, commercialized as Parsol $\mathrm{MCX}^{\mathrm{TM}}$ ), which, upon UV-irradiation, undergo E/Zisomerization via the excited triplet state. In the special case of ortho-hydroxy cinnamates (coumarates), $\mathbf{1}$, the $Z$-isomer is unstable because of the spatial proximity of the ester group and the phenolic hydroxyl group, leading to fragmentation of the molecule into coumarin 3 (which may carry additional substituents) and the alcohol moiety (Scheme 1).

This potentially very useful transformation has been scarcely mentioned in the literature. The first study was reported in 1934 by Dey et al., ${ }^{[2]}$ who found that lightinduced lactonization was much cleaner and faster than the thermally induced process. E-coumarates as photolytically removable 'blocking' groups for thrombine and other enzymes have been reported by Porter and coworkers. ${ }^{[3]}$ Wang and coworkers studied $Z$-coumarates as lipase-cleavable protecting groups for alcohols, ${ }^{[4]}$ whereas $E$-o-hydroxy cinnamic amides have been proposed 


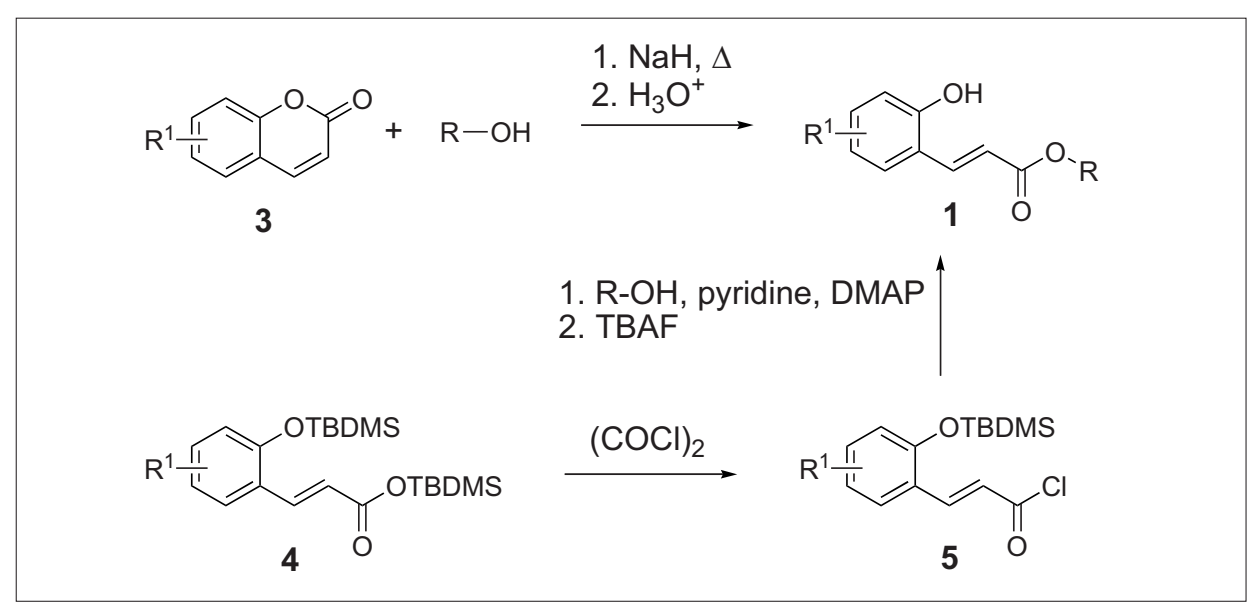

Scheme 2. Preparation methods of $E$-coumarates

by the same group as light-cleavable protecting groups for amines. ${ }^{[5]}$

We became interested in $E$-coumarates in the context of controlled fragrance release, since these compounds offer the unique possibility of delivering coumarin and a fragrance alcohol from a single precursor structure with no additional weight. ${ }^{[6]}$ Coumarin is a highly appreciated fragrance ingredient used in many different olfactory themes, but lacks substantivity in rinse-off applications. The same holds true for many valuable perfumery alcohols and phenols.

For the preparation of perfumistically interesting $E$-coumarates, we have employed two main synthetic routes which are summarized in Scheme 2.

A few entries in the literature on the direct reaction of coumarin with large excesses of sodium methoxide, e.g. ${ }^{[7]}$ to yield the corresponding methyl $E$-coumarates, have prompted us to explore this reaction with sodium salts of perfumery alcohols. We were pleased to find that simply reacting coumarin with 1-2 equiv. of the sodium salt of primary, secondary and even tertiary perfumery alcohols in a variety of non-protic solvents furnished, after hydrolysis, the desired $E$-coumarates in useful to excellent yields. Hence, in terms of materials, preparation of and fragrance release from $E$-coumarates are symmetrical to each other, the former being a thermal, alkalipromoted process, the latter a photochemical one, but both featuring an E/Z-double bond isomerization as the key element. Unfortunately, the small but noble group of phenolic perfumery raw materials, such as vanillin or eugenol, does not react with coumarin to form $E$-coumarates. Therefore, we developed a second route, which starts from commercially available $E$-coumaric acid. After bis-silylation, ester $\mathbf{4}$ is transformed into acid chloride $\mathbf{5}$ and then coupled with the phenolic fragrance ingredient. The final deprotection step requires carefully controlled conditions in order to avoid saponification of the fragile phenol esters.
The isomerization and fragmentation of a large series of E-coumarates under irradiation in a photoreactor was monitored in a time-resolved manner. We found that reaction rates are, as expected, highly structure dependant. Four typical examples are represented in Fig. 1.

Under the relatively harsh irradiation conditions employed, the transformation of $E$-coumarates of primary alcohols into coumarin and the alcohol generally reaches completion within 10-20 min, as exemplified by the irradiation of 9-decenyl- $E$-coumarate $\left(6, \lambda_{\max } 326 \mathrm{~nm}, \varepsilon\right.$ 135'914). Irra-

diation of its 7'-methoxy analogue 7 leads to a rapidly decreasing conversion rate with build up of Z-7. This might be due to the reduced electrophilicity of the ester group, but also of a possible interference of the product, 7-methoxycoumarin (methylumbelliferon), with the photoisomerization of the starting material (internal quenching of excited states). In the case of the corresponding $o$ $t$-butyl derivative $\mathbf{8}$, the lactonization rate is reduced, most probably as a result of the steric compression occurring upon attack of the phenolic hydroxyl group to the ester to form a tetrahedral intermediate ('buttressing effect' ${ }^{[8]}$ ). Finally, the dependence of the rate of saponification or transesterification on the steric bulk of the alcohol moiety to be exchanged has been described and rules established (e.g. Newman's empirical 'rule of six' $\left.{ }^{[9]}\right)$. Thus, upon irradiation of the dihydromyrcenyl coumarate 9 for 30 min., a mixture is formed containing $57 \% \mathrm{Z}$ - and only $34 \%$ E-coumarate (a roughly $2: 1$ ratio of $Z: E$ in the photoequilibrium state has also been found for other slowly- or non-lactonizing coumarates). Lactonization of an isolated sample of the $Z$-isomer kept away from light takes a full week.

We are thus able to control the rate of perfume release over a wide range of time through proper choice of the bulkiness of the attached fragrance alcohol.

During these studies, we also recorded preliminary data showing a negative influ-

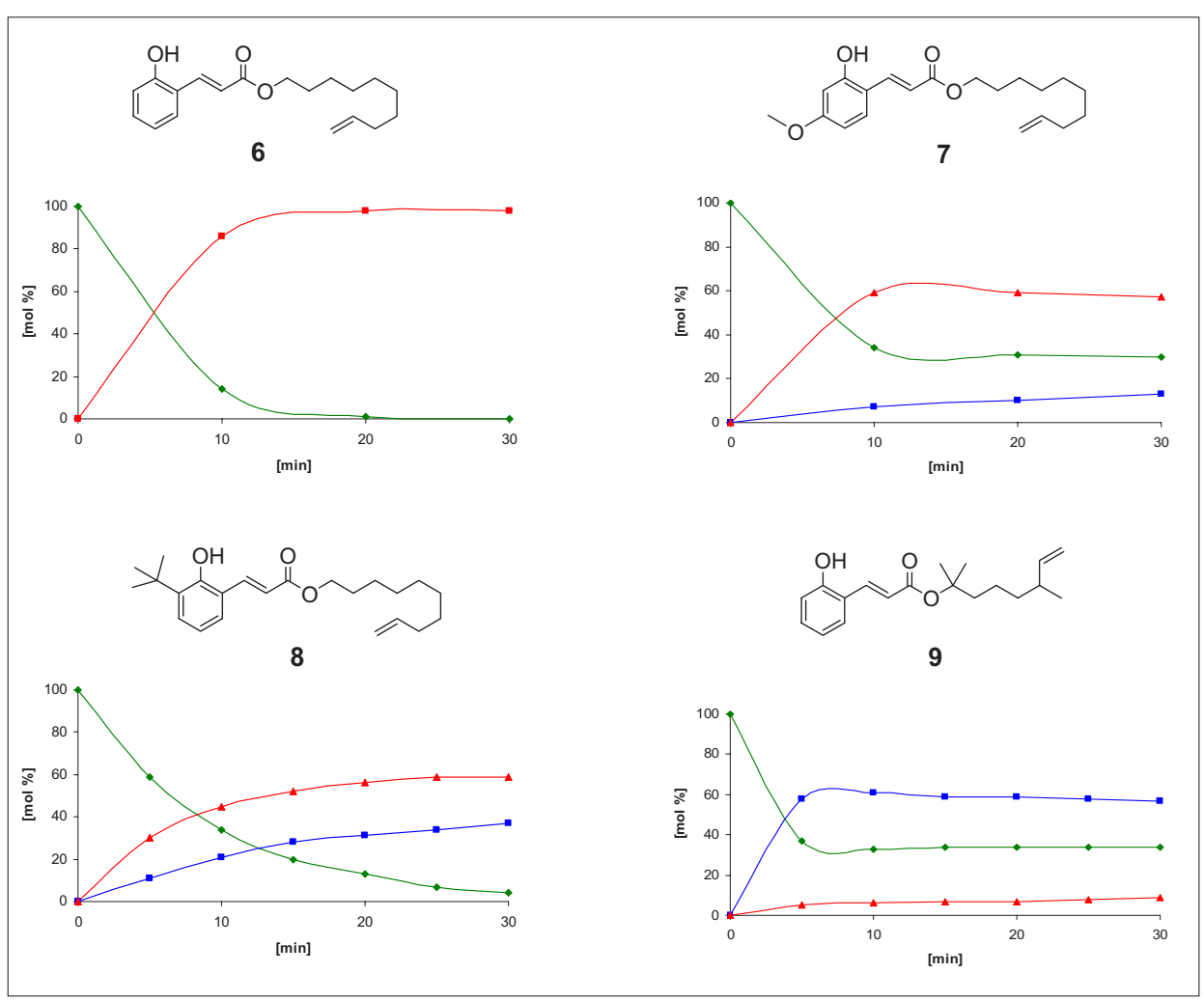

Fig. 1. Photoisomerization/lactonization of different $E$-coumarates $\left(0.5 \mathrm{mM}\right.$ solutions in $\mathrm{CH}_{3} \mathrm{CN} / \mathrm{H}_{2} \mathrm{O}$ 9:1, irradiation in a Pyrex ${ }^{\circledR}$ photoreactor equipped with a $150 \mathrm{~W}$ mercury medium pressure lamp, analysis by RP-HPLC). Green: E-coumarate Blue: Z-coumarate Red: (substituted) coumarin. 
ence of UV-irradiation on the lactonization rates. The underlying reason might be the well-documented photoenhanced acidity of the phenolic $\mathrm{OH}$-group, ${ }^{[10]}$ leading to a less nucleophilic phenolate. Additional studies on this rather unexpected additional influence of light on perfume release are currently under way.

\section{Fragrance Precursors Based on the Norrish Type II Photo-Cleavage}

The first Norrish type II-based fragrance precursor system was devised by Anderson and Fráter in 1998.[11] Other systems based on the acetophenone motive ${ }^{[12]}$ and $\alpha-k e-$ toesters ${ }^{[13]}$ have been studied by Herrmann and coworkers. This family of $\alpha$-alkoxyacetophenones $\mathbf{1 0}$ releases upon UV-light irradiation acetophenones and either aldehydes or ketones depending on the nature of the odiferous species to be released from the alkoxy part (Scheme 3). A more detailed description of the release mechanism is given below. The synthesis of $\mathbf{1 0}$ is straightforward, starting with the alkoxylation of bromoacetonitrile with either a primary or a secondary alcohol derived from a fragrant aldehyde or ketone respectively to yield the nitrile 11. This is then followed by the addition of a phenylmagnesium halide and the hydrolysis of the so-obtained imine. Evidently, the selection of the substitution pattern of the benzene ring governs the chromophoric properties of the fragrance precursor system and thus the potential ease of cleavage. It is obviously desirable that both fragments resulting from the Norrish type II cleavage, namely the acetophenone part and the aldehyde or ketone, be well-established perfumery chemicals. However, in order to optimize the photochemical performance of the precursor, it may be more desirable to choose a non-odoriferous chromophore

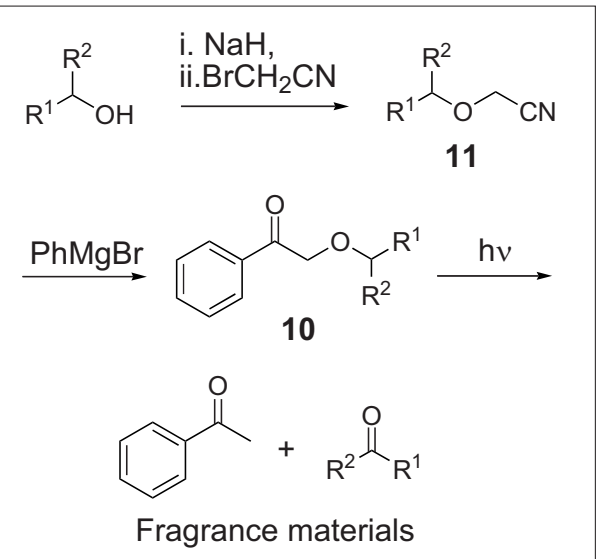

Scheme 3. Synthesis of fragrance precursors 10 and photochemical odorant release. Substituents on the aromatic rings are omitted for the sake of clarity.

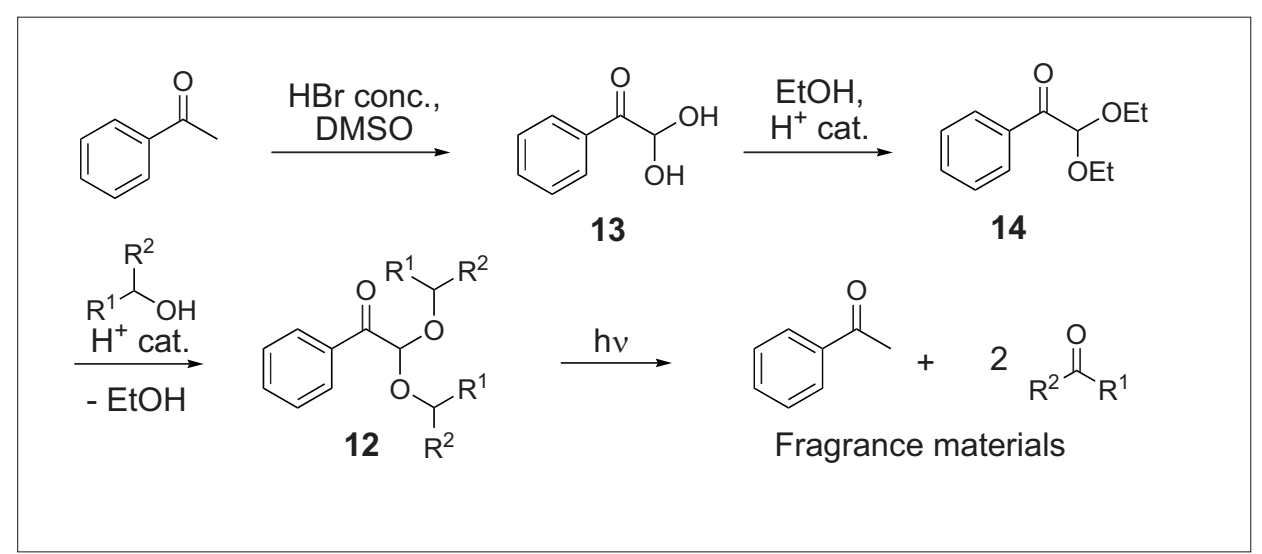

Scheme 4. Synthesis of fragrance precursors 12 and photochemical odorant release. Substituents on the aromatic rings are omitted for the sake of clarity.

and concentrate the fragrant aspect on the carbonyl compound to be released.

In this light, the system has subsequently been further tuned to release two carbonyl compounds from one chromophore. ${ }^{[14]}$ The resulting $\alpha, \alpha$-dialkoxyacetophenone structures 12, a simple representative being the diethoxyacetophenone, are well known and used as starter for photoinitiated radical polymerization. ${ }^{[15]}$ Given the constraint that a light-labile profragrance is expected to cleave at natural or even artificial light with a limited UV proportion, as opposed to intense mercury lamps or UV lasers used for the curing of monomers, a simple acetophenone was not entirely suitable as chromophore. Thus, a series of more electron-rich benzene rings with a bathochromic shift was employed: 4-alkoxy- $\left(\lambda_{\max }\right.$ approx. $+30 \mathrm{~nm}$ $v s$ acetophenone), 3,4-dialkoxy- $(+60 \mathrm{~nm})$, 4-acylamino- $(+45 \mathrm{~nm})$, and 4-dialkylamino- (+98 nm) substituted acetophenones. The synthesis of these $\alpha, \alpha$-dialkoxyacetophenones 12 differs significantly from the above-described ethers 10, in which it usually starts from the acetophenone. These are oxidized to the corresponding hydrated arylglyoxal 13, using the protocol of Floyd et al., involving hydrobromic acid and dimethyl sulfoxide. ${ }^{[16]}$ Although not absolutely necessary, but more convenient for the reason of solubility, the intermediate $\mathbf{1 3}$ is then first converted to the diethyl acetal 14, from which the fragrance precursors are accessible via trans-acetalization with the required fragrance aldehyde or ketone-derived alcohols (Scheme 4 ).

In the course of this research, it became desirable not only to release ketones and aldehydes, but also esters and lactones, which are included among the most important fragrance materials, especially for fruity accords. Owing to the wide scope of the Norrish type II reaction, the most evident development was the substitution of the beta-carbon atom in the skeleton $\mathbf{1 0}$ for an oxygen, in order to give rise to esters upon cleavage. Thus, the phenacyl acetals $\mathbf{1 5}$ are light-labile precursors for odoriferous esters $^{[17]}$ and, accordingly, the respective cyclic structures $\mathbf{1 6}$ for lactones. ${ }^{[18]}$ The synthesis of these species as well as their cleavage is shown in Scheme 5. Key intermediate is the $\alpha$-hydroxy-ketone 17, prepared from the corresponding acetophenone by $\alpha$-bromination, formylation with sodium formate in aqueous ethanol and subsequent hydrolysis. The alcohol $\mathbf{1 7}$ is then treated with either linear or cyclic enol ethers under the catalytic action of trifluoroacetic acid, to afford the required phenacyl acetals $\mathbf{1 5}$ or $\mathbf{1 6}$, respectively.

The fragrance release mechanism (photolysis) of the above-described properfumes is exemplified on the Lilial ${ }^{\circledR}$-precursor 18, with the chromophore being $p$-propoxyacetophenone ( $\lambda_{\max } 278 \mathrm{~nm}, \varepsilon$ 17600). It has a very faint odor which does not disturb the scent quality of Lilial ${ }^{\circledR}$ upon release. In the expected Norrish type II fragmentation (Scheme 6$)$, the ground state $\left(S_{0}\right)$ carbonyl group is excited to the $S_{1}$ state, upon which an intersystem crossing gives rise to the triplet carbonyl $\mathrm{T}_{1}$, from which $\gamma$-hydrogen abstraction (or 1,5-H shift) takes place. ${ }^{[19]}$ At this stage, two competing reactions are occurring: on the one hand, the $\beta$-scission required for Lilial $^{\circledR}$ release, and on the other hand, the intramolecular recombination of the diradical $\mathbf{1 9}$ to the corresponding oxetane 20. An experiment (Fig. 2), in which a $0.1 \%$ solution of the precursor $\mathbf{1 8}$ in acetonitrile is irradiated with a mercury medium pressure lamp $(150 \mathrm{~W})$ in a borosilicate $\left(\right.$ Pyrex $^{\circledR}$, cut off at approximately $300 \mathrm{~nm}$ ) apparatus over a period of $40 \mathrm{~min}$, demonstrated that the two competing reactions occur to a similar extent (analysis by HPLC of samples taken every $5 \mathrm{~min}$ ) and that the rate of recovery (yield) lays in the order of $45-60 \%$.

Evidently, the Norrish type II reaction is not the only possible reaction to occur upon irradiation. The $\alpha$-fragmentation, also 

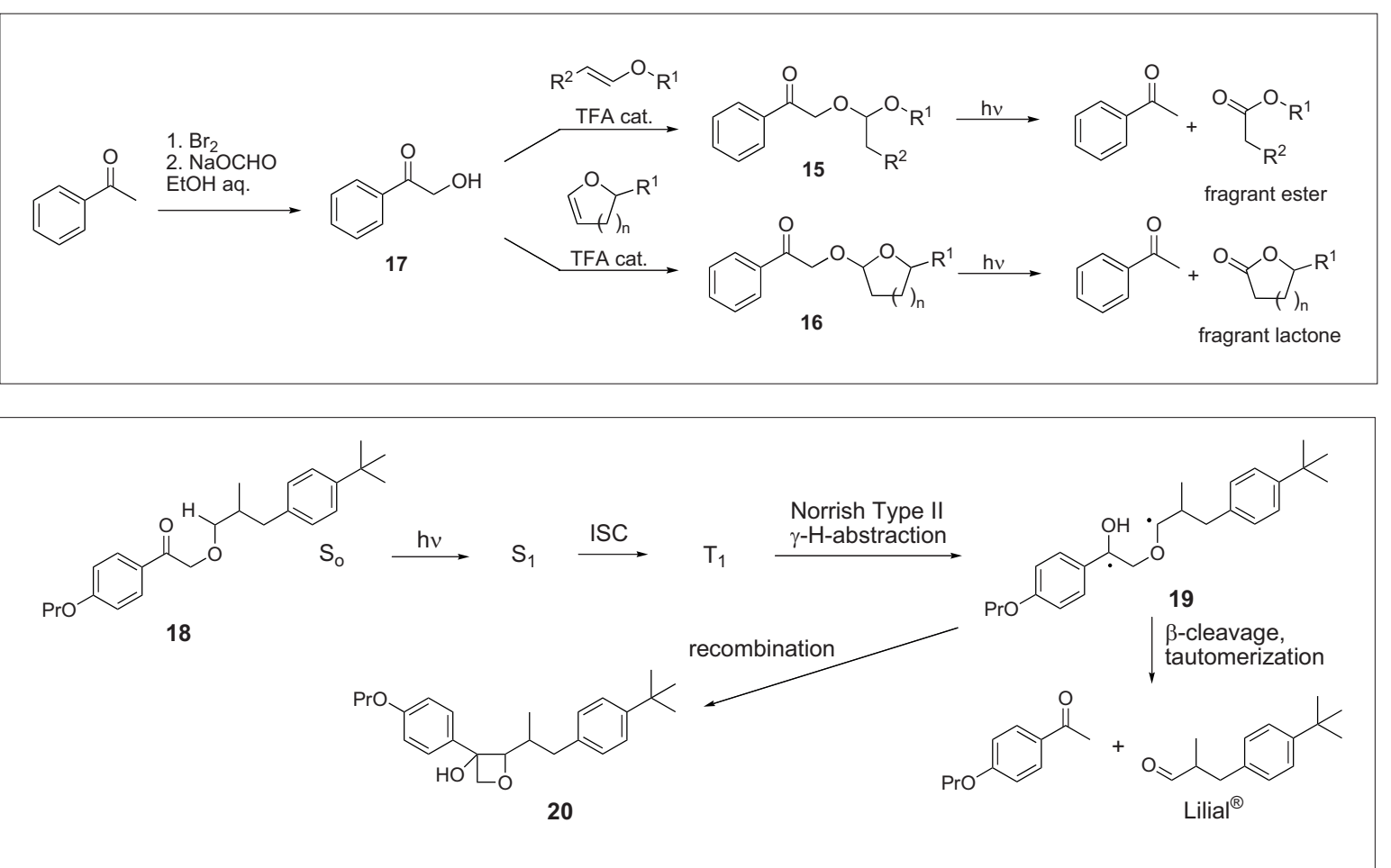

Scheme 5. Synthesis of fragrance precursors 15 and 16 and photochemical release of fragrant esters and lactones. Substituents on the aromatic rings are omitted for the sake of clarity.

Scheme 6 .

Photofragmentation of Lilial ${ }^{\circledR}$-precursor 18

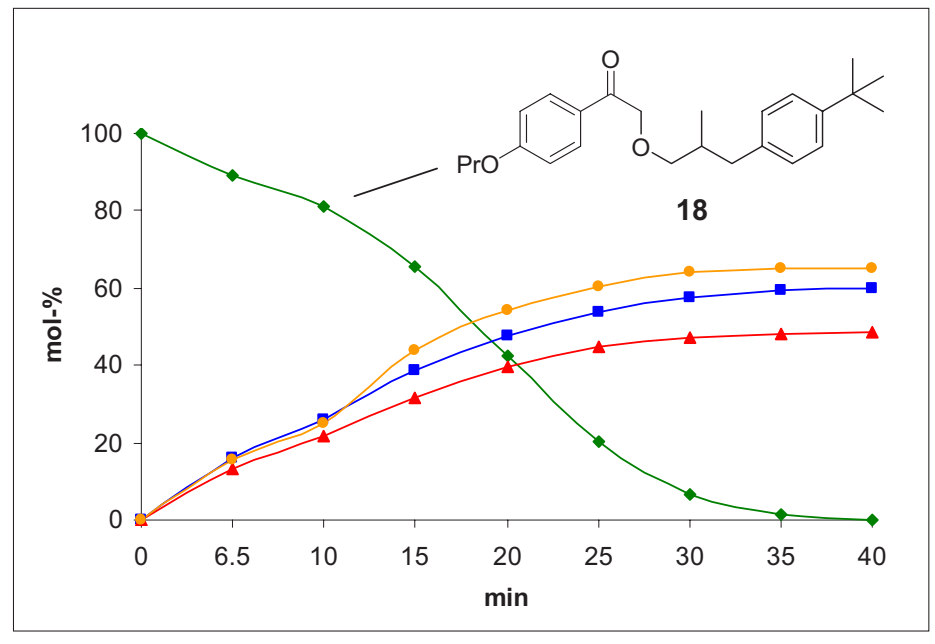

Fig. 2. Kinetic study of the photorelease of Lilial $^{\circledR}$ (blue), 4-propoxyacetophenone (red) and Oxetane $\mathbf{2 0}$ (yellow) from their precursor 18

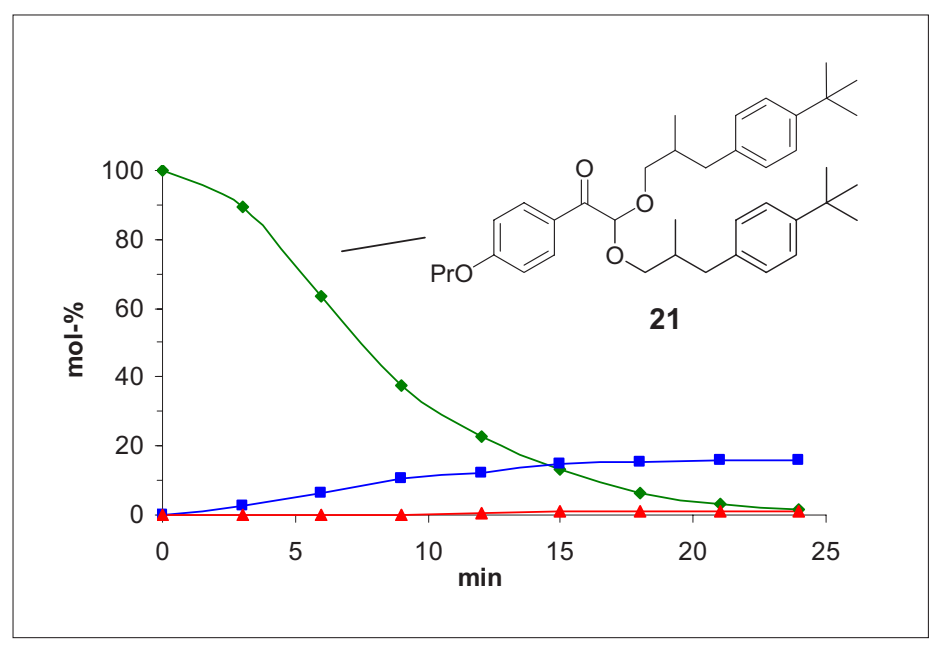

Fig. 3. Kinetic study of the photorelease of Lilial $^{\circledR}$ (blue) and 4-propoxyacetophenone (red) from their precursor 21 termed Norrish type I cleavage, is also expected in such compounds. ${ }^{[15]}$ This is observed especially with the $\alpha, \alpha$-dialkoxyacetophenones $\mathbf{1 2}$, and the corresponding, relatively long lived benzoyl and dialkoxymethyl radicals, are believed to be the reason for their good performance as photoinitiators in the radiation curing of e.g. acrylate monomers. ${ }^{[20]}$ For the fragrance precursor purpose, this $\alpha$-fragmentation is, of course, absolutely undesirable as it does not give rise to the required odorants. Performing an analogous kinetic experiment with the Lilial ${ }^{\circledR}$-precursor $21\left(\lambda_{\max } 285\right.$ $\mathrm{nm}, \varepsilon$ 24000), offered some evidence for competing mechanisms (Fig. 3). Although no products related to the fragments from the $\alpha$-scission have been isolated and characterized, the rather low rate of recovery of the required aldehyde (15\%) and acetophenone $(2 \%$ !) are an indication of an unwanted photodegradation of either the precursor or the products or both. In addition, the expected intermediate mono-alkoxy-acetophenone 18 was not observed. This leads to the hypothesis that the photolysis of the latter is significantly faster than that of the dialkoxyacetophenone 21. Nevertheless, application tests have demonstrated that the release of Lilial ${ }^{\circledR}$ from its precursor $\mathbf{2 1}$ is sufficient to obtain an olfactory signal that, in terms of longevity, can easily compete with the effect of the free fragrance material. It should thus be noted that the laboratory irradiation conditions are rather harsh compared to natural sunlight.

\section{Fragrance Precursors Based on Xanthate Esters}

As part of our efforts to find alternative photocleavable fragrance precursors, we also envisaged that xanthenoic esters may release fragrant alcohols, based on a disclosure of Barton et al., who observed the photolytic cleavage of xanthenoic aryl esters into xanthene and the corresponding phenols. ${ }^{[21]}$ We expected that such esters would be capable of releasing fragrant alcohols by a homolytic cleavage of the $\mathrm{CO}-\mathrm{OAr}$ bond, according to the well precedented photochemical Fries rearrangement, ${ }^{[22]}$ followed by decarbonylation. Not quite unexpectedly, however, we have discovered that only a small amount of alcohol is formed from alkenyl xanthenoic esters 22 (Scheme 


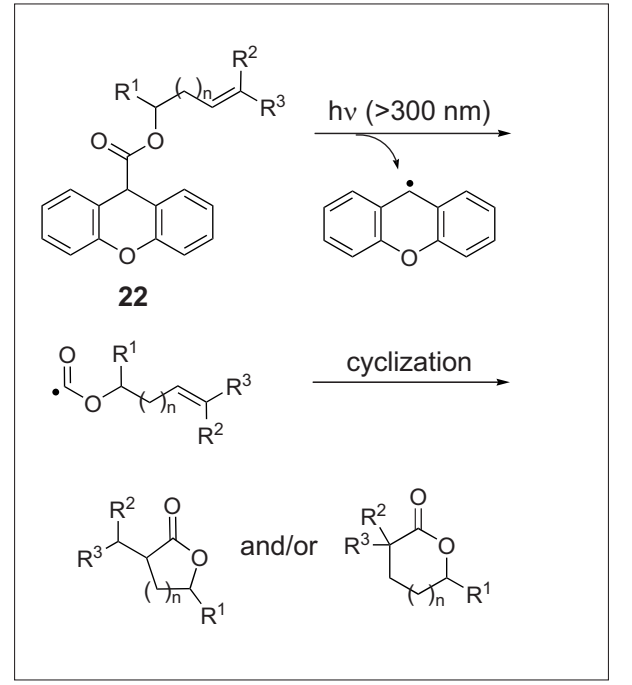

Scheme 7. Photochemical reaction of xanthenoic esters 22 to produce lactones as major products

7). The major products were found to be lactones of various ring sizes, depending on the location of the unsaturation in the parent alcohols. ${ }^{[23]}$ It is fair to say that for both economical reasons and their limited performance, these xantenoic esters have little potential as commercial fragrance precursors. Therefore, our efforts have not proceeded beyond the 'proof of concept' stage.

\section{Conclusion}

As a novel approach to improve the longevity of perfumes, this account describes three different photo-labile fragrance precursor types for the release of selected fragrance materials. Homolytic bond cleavages from photoexcited radical species occur in $\alpha$-alkoxyacetophenones and xanthate esters. The former lead to the efficient release of perfumery aldehydes, whereas fragrant lactones of various ring sizes are released from the latter via cyclization of intermediate acyl radical species onto internal olefins. Mild irradiation conditions induce an $E / Z$-double bond isomerization in $E$-coumarates, followed by clean and efficient release of coumarin and a fragrance alcohol via a spontaneous intramolecular transesterification.

The materials described in this overview offer not only the desired slow and/or timed release of fragrance ingredients, but as in the case of the $\alpha$-alkoxyacetophenones, are also a means of protecting fragile perfume ingredients, such as aldehydes, which are prone to oxidation in harsh consumer product bases (e.g. detergents). Moreover, profragrances are more substantive than the free fragrance materials, resulting in better deposition on the target support, such as fabric in laundry care, skin and hair in personal care or hard surfaces in the application of all purpose cleaning.

Thus, our examples demonstrate how the use of photochemistry adds a whole new creative and technical dimension to the traditional ways of fragrancing. As the first commercial application of this research, the coumarin/Rosalva precursor 6 has been recently introduced into the perfumer's palette under the name of Tonkarose $\mathrm{T}^{\mathrm{TM}}$.

Received: July 27, 2007

[1] For overviews on modern fragrance ingredients: a) G. Fráter, J. Bajgrowicz, P. Kraft, Tetrahedron 1998, 54, 7633; b) P. Kraft, J. Bajgrowicz, C. Denis, G. Fráter, Angew. Chem., Int. Ed. 2000, 39, 2980.

[2] B. B. Dey, R. H. R. Rao, T. R. Seshadri, J. Ind. Chem. Soc. 1934, 743.

[3] a) A. D. Turner, S. V. Pizzo, G. W. Rozakis, N. A. Porter, J. Am. Chem. Soc. 1987, 109, 1274; b) A. D. Turner, S. V. Pizzo, G. W. Rozakis, N. A. Porter, J. Am. Chem. Soc. 1988, 110, 244; c) N. A. Porter, J. W. Thuring, H. Li, J. Am. Chem. Soc. 1999, 121, 7716.

[4] a) B. Wang, J. Zhang, W. Wang, Bioorg. Med. Chem. Lett. 1996, 6, 945; b) Y. Liao, S. Hendrata, S. Y. Bae, B. Wang, Chem. Pharm. Bull. 2000, 48, 1138.

[5] B. Wang, A. Zheng, Chem. Pharm. Bull. 1997, 45, 715 .

[6] a) G. Fráter, D. Anderson, US 6096918, priority 13.2.1998, to Givaudan SA; Chem. Abstr. 131, 170173; b) F. Flachsmann, J.P. Bachmann, WO 2005077881, priority 12.2.2004, to Givaudan SA; Chem. Abstr. 143, 229566.

[7] N. Cairns, L.M. Harwood, P. D. Astles, Chem. Commun. 1986, 16, 1264.

[8] a) M. Rieger, F. H. Westheimer, J. Am. Chem. Soc. 1950, 72, 19; b) for a modern example: K. Maeda, Y. Okamoto, O. Toledano, D. Becker, S. E. Biali, Z. Rappoport, J. Org. Chem. 1994, 59, 5473.

[9] M. S. Newman, J. Am. Chem. Soc. 1950, 72,4783 .

[10] a) C. Sandorfy, Compt. Rend. 1951, 232, 841; b) H. Konschin, R. Lunnala, F. Sundholm, Suomen Kemistiseuran Tiedonantoja 1973, 82, 8. c) S. C. Shim, J. W. Park, H. S. Ham, J. S. Chung, Bull. Korean Chem. Soc. 1983, 4, 45 .

[11] D. Anderson, G. Fráter, EP 983990, priority 4.9.1998, to Givaudan SA; Chem. Abstr. 132, 194193.

[12] a) B. Levrand, A. Herrmann, Flav. Frag. J. 2006, 21, 400. b) A. Herrmann, WO 2001096272, priority 11.06 .2000 , to Firmenich SA.

[13] a) S. Rochat, C. Minardi, J.-Y. De Saint Laumer, A. Herrmann, Helv. Chim. Acta 2000, 83, 1645; b) A. Herrmann, C. Debonneville, V. Laubscher, L. Aymard, Flav. Frag. J. 2000, 15, 415. c) A. Herrmann, US 6218355 , priority 21.05 .1999 , to Fir- menich SA; Chem. Abstr. 134, 300652.

[14 ] S. Derrer, M. Gautschi, EP 1262473, priority 30.5.2001, to Givaudan SA; Chem. Abstr. 138, 8276

[15] J. E. Christensen, A. F. Jacobine, C. J. V. Scanio, Radiation Curing 1981, $8,4$.

[16] M. B. Floyd, M. T. Du, P. F. Fabio, L. A. Jacob, B. D. Johnson, J. Org. Chem. 1985, $50,5022$.

[17] M. Gautschi, C. Plessis, S. Derrer, EP 1146033 , priority 10.4 .2000 , to Givaudan SA; Chem. Abstr. 135, 308610.

[18] M. Gautschi, C. Plessis, S. Derrer, EP 1167362 , priority 19.6 .2000 , to Givaudan SA; Chem. Abstr. 136, 74334.

[19] P. J. Wagner, Acc. Chem. Res. 1971, 4, 168.

[20] J. P. Fouassier, D. J. Lougnot, J. Chem. Soc., Faraday Trans. 1 1987, 83, 2935.

[21] D. H. R. Barton, Y. L. Chow, A. Cox, G. W. Kirby, J. Chem. Soc. 1965, 3571.

[22] H. Kobsa, J. Org. Chem. 1962, 27, 2293.

[23] C. Plessis, S. Derrer, Tetrahedron Lett. 2001, 42, 6519. 